\title{
A case of iodide mumps following contrast CT imaging; an exploration of its pathogenesis
}

\author{
Aravinthan $\mathbf{M}^{1}$, Jayasinghe $\mathrm{S}^{1,2}$ \\ ${ }^{I}$ Professorial Medical Unit, National Hospital of Sri Lanka, Colombo, Sri Lanka. \\ ${ }^{2}$ Department of Clinical Medicine, Faculty of Medicine, University of Colombo, Sri Lanka. \\ Correspondence: Dr. Mythily Aravinthan \\ e-mail: mailmythi@gmail.com \\ D https://orcid.org/0000-0002-2335-8553 \\ Submitted on 09.05.2021 and accepted for publication on 02.08.2021
}

\section{Introduction}

Iodine containing contrast media are widely used in diagnostic and therapeutic procedures like angiography, contrast-enhanced computed tomography (CT) and angioplasties. The adverse events to contrast media range from allergic reaction to anaphylaxis and contrast nephropathy. 'Iodide Mumps' refers to benign swelling of the salivary glands following the use of iodide contrast which was first described in 1956 by Sussman and others from Israel (1). Since then, over seventy-five cases of the same have been reported (2). But its incidence is postulated at $1-2 \%$ (3). Awareness of this rare event and its benign course helps avoid unnecessary anxiety for the patient and the attending medical team.

\section{Case Presentation}

A 64-year-old male with type 2 diabetes mellitus, hypertension, chronic kidney disease (CKD) stage III (eGFR - $34 \mathrm{ml} / \mathrm{min} / 1.73 \mathrm{~m}^{2}$ ) and Child-Pugh B alcoholic chronic liver cell disease underwent a contrast enhanced Computed Tomography (CT) scan to evaluate a suspicious lesion of the liver. The procedure involved the use of $100 \mathrm{ml}$ of low osmolar non-ionic iodide contrast ioxehol (Omnipaque $300 ; 300 \mathrm{mg} / \mathrm{ml}$ iodide). Around 8 hours after the procedure he noted a rapid painless swelling of his neck. The patient was highly anxious but denied any difficulty in swallowing or breathing.
Clinical evaluation revealed an afebrile patient with bilateral symmetrical non tender enlargement of the submandibular salivary glands without erythema or warmth (Figure 1). His vital signs were stable and there were no signs of angioedema or anaphylaxis. Basic laboratory investigations were within the normal range $(\mathrm{WBC}-7000 / \mu \mathrm{l} ; \mathrm{Hb}-11 \mathrm{~g} / \mathrm{dl}$; platelets - $170 \times 10^{3} / \mu \mathrm{l} ; \mathrm{CRP}<6 \mathrm{mg} / \mathrm{dl} ;$ ESR -18 $\mathrm{mm} / \mathrm{hr}$; serum potassium - $4.8 \mathrm{mmol} / \mathrm{l}$; serum sodium - $139 \mathrm{mmol} / \mathrm{l}$; serum creatinine - $1.8 \mathrm{mg} / \mathrm{dl}$ ). An ultrasound scan of the neck confirmed diffusely enlarged submandibular glands with milder enlargement of bilateral parotids without any calculi or other abnormalities involving the thyroid or lymph nodes. There was no evidence of facial nerve palsy. Trauma and other viral or bacterial infectious causes were excluded by history, normal inflammatory markers and infection screen (mumps/ EBV and HIV serology). With the available findings a clinical-radiological diagnosis of 'iodide mumps' was made. Oral paracetamol $1 \mathrm{~g}$ as and when needed was given for the mild discomfort which he complained subsequently. He was kept under observation for 24 hours for delayed reactions and was discharged with a follow up at two weeks. The swelling had started to reduce by two days and was completely resolved at two weeks. 


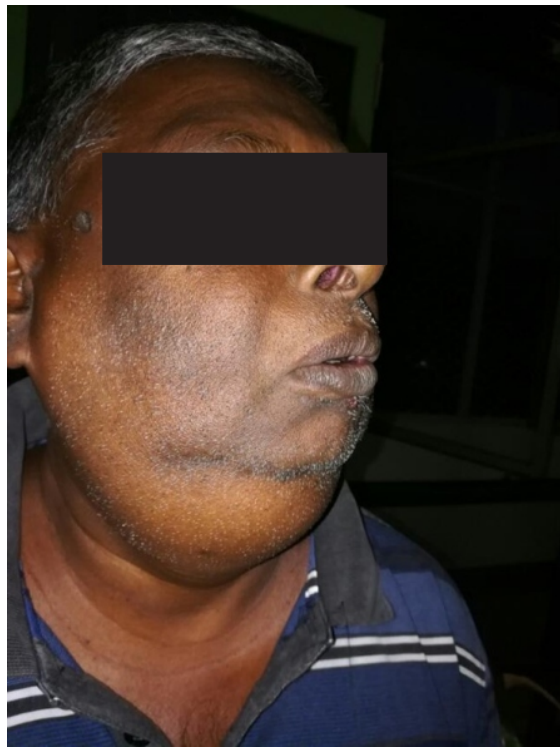

Figure 1: Bilateral submandibular swelling

\section{Discussion}

Circulating Iodide is concentrated in the thyroid gland and all the exocrine secretions containing chloride ions including saliva, sweat, tears, gastric and pancreatic juices. Iodide enters these sites via the sodium-iodine symporter; an intrinsic membrane protein and is thought to have important biological roles in the form of an anti-oxidant and local mucosal protective agent (4). Under normal circumstances, most iodide is excreted unchanged by the kidneys and the remainder via the above secretions. Although the exact pathogenesis is unclear, it is postulated that high concentrations of iodide can cause local mucosal oedema and ductal obstruction leading to a non-inflammatory swelling of the salivary glands $(3,5)$. Submandibular and Parotid are the most affected but thyroiditis and pancreatitis can also occur $(2,6,7)$. Iodide mumps is different from 'iodism' which refers to chronic dose dependent systemic iodine toxicity (1). Iodism is more severe and involves systemic features like fever, anorexia, cachexia, nausea, vomiting, skin lesions known as iododerma and inflammation of respiratory and gastrointestinal mucosae including salivary glands. Iodism can be fatal whereas iodide mumps refers to the acute idiosyncratic manifestation which is benign (1).

The contrast in this case, iohexol, is a non-ionic water-soluble iodine compound that is excreted unaltered, predominantly through the kidneys. Following rapid intravenous injection, peak blood levels occur immediately followed by a rapid fall within five to ten minutes and the vascular compartment half-life is around twenty minutes. Approximately $90 \%$ of injected iodide is excreted in urine within the first 24 hours (8). The urinary and biliary excretion possibly affected by coexistent CKD and cirrhosis could have led to the delay of higher peak levels of the drug occurred accompanied by prolonged half-life. There is little data on the concentration of iohexol in the salivary gland. However, it is reported that concentration of iodine in salivary glands is 30 times of that of plasma (8).

According to a meta-analysis, age, osmolarity of contrast, volume of contrast or the renal function do not influence the occurrence of this reaction and it is idiosyncratic in nature. The onset of symptoms can be immediate or delayed maybe up to 5 days. Often it is a painless swelling resolving without sequelae as in our case. Tenderness is seen in up to $49 \%$ with few experiencing sense of choking and swallowing difficulty (2). Facial nerve palsy secondary to parotid swelling is by far the most sinister sequelae reported (2).

Symptoms completely resolve over a median duration of 3 days with a delay of up to 72 days in few cases (2). Prophylactic and therapeutic interventions using steroids, non-steroidal antiinflammatory agents, antihistamines, saline gargle, warm soaks and dialysis have no influence on the occurrence or the time to recovery (10). Older age and longer time to onset of symptoms haven been associated with delayed resolution of swelling (2).

The stereotypic reaction is known to recur with repeated exposure to iodide contrast sometimes with increasing severity but has been well tolerated without any sequelae (3). It can occur in those without any known allergy and can occur de-novo in those who have previously tolerated iodide contrast uneventfully (3).

\section{Conclusions}

'Iodide mumps' is a benign, obvious, under-diagnosed complication of post-iodide contrast media exposure. This can be diagnosed clinicalradiologically. Despite causing discomfort and intense anxiety, reassurance alone suffices avoiding un-necessary interventions. 
Authors disclose no conflicts of interest. The written informed consent has been obtained from the patient to publish this case report with photographs.

\section{References}

1. Sussman RM, Miller J. Iodide mumps after intravenous urography. NEngl JMed. 1956; 255(9): 433-434.

2. Jiao A, Farsad K, McVinnie DW, Jahangiri Y, Morrison JJ. Characterisation of iodide-induced sialadenitis: metaanalysis of the published case reports in the medical literature. Acad Radiol. 2020; 27(3): 428-435.

3. Egan M, Maglione PJ. Multiple reasonably tolerated percutaneous coronary interventions in a patient with iodide mumps. Ann Allergy, Asthma Immunol Off Publ Am Coll Allergy, Asthma, Immunol. 2015; 115(3): 253-254.

4. Venturi S, Venturi M. Iodine in evolution of salivary glands and in oral health. Nutr Health. 2009; 20(2): 119-134.

5. Zhang G, Li T, Wang H, Liu J. The pathogenesis of iodide mumps: a case report. Medicine (Baltimore). 2017;96(47)
6. Gilgen-Anner Y, Heim M, Ledermann H-P, Bircher AJ. Iodide mumps after contrast media imaging: a rare adverse effect to iodine. Ann Allergy, Asthma Immunol. 2007; 99(1): 93-98.

7. Moisey RS, McPherson S, Wright M, Orme SM. Thyroiditis and iodide mumps following an angioplasty. Nephrol Dial Transplant. 2007; 22(4): 1250-1252.

8. General-Electric-Company. OMNIPAQUE ${ }^{\mathrm{TM}}$ (iohexol) Injection [Internet]. 2017. Available from: https://www.accessdata.fda.gov/drugsatfda_docs/label/20 17/018956s099lbl.pdf

9. Kohri K, Miyoshi S, Nagahara A, Ohtani M. Clinical Note: Bilateral Parotid Enlargement ("Iodide Mumps") Following Excretory Urography. Radiology. 1977; 122(3): 654.

10. Panasoff J, Nusem D. Iodide mumps. World Allergy Organ J. 2008; 1(5): 85-86 